\title{
E. Mari, O. Trukhanova, M. Valeri (a cura di), Un radioso avvenire? L'impatto della Rivoluzione d'Ottobre sulle scienze umane/Svetloe budušcee? Itogi i perspektivy Ioo let spustja, Edizioni Nuova Cultura, Roma 2019, pp. 527.
}

Il volume curato da Emilio Mari, Olga Trukhanova e Marta Valeri raccoglie gli atti di due convegni internazionali organizzati in occasione del centenario della Rivoluzione d'Ottobre.

Si tratta di un importante progetto, ideato da Ornella Discacciati e Claudia Scandura, che ha visto il coinvolgimento della Sapienza Università di Roma, dell’Università degli Studi della Tuscia, del Museo Laboratorio di Arte Contemporanea (MLAC) di Roma, della Facoltà di Storia dell'Università Statale di Mosca e dell'Istituto dell'Arte Realistica di Mosca (IRRI), con il patrocinio dell' Istituto Italiano di Cultura a Mosca. Il primo convegno, Rossija-Italija: 'Realizmy'v iskusstve i kul'ture XX veka (Russia-Italia: 'Realismi' nell'arte e nella cultura del XX secolo) si è svolto a Mosca il 24-25 novembre 2016; il secondo convegno, che dà il titolo al volume, si è svolto a Roma il 6-8 novembre 2017. Ad entrambi gli eventi hanno partecipato autorevoli studiosi provenienti da università e da centri di ricerca internazionali, i quali hanno apportato preziosi contributi alla discussione critica sulla Rivoluzione d'Ottobre, secondo una prospettiva multidisciplinare nell'ambito delle scienze umane, affrontando temi legati a letteratura, storia, moda, arte, cinema e teatro, economia e società.

È impossibile rendere conto di tutti i saggi che compongono quest'opera ricca ed eclettica. Vorrei, tuttavia, soffermarmi su due momenti di riflessione ispirati dal suo titolo.

Il primo riguarda la 'luce' che si diffonde dall'aggettivo 'radioso', collegato all'idea di futuro, e che i curatori mettono emblematicamente in relazione con la complessa opera di Marc Chagall Révolution. Il quadro, che l'autore iniziò a dipingere nel 1937, per ritornarci a più riprese, fino al suo completamento nel 1968 sotto l'influsso del maggio francese, ha tre punti di luce che si definiscono in crescendo all'occhio dell'osservatore. La luce è rappresentata dalla fioca candela che illumina la strada a chi si è incamminato verso una vita nuova, dalla lampada che rischiara il tavolo a cui Lenin, come un equilibrista, si appoggia a testa ingiù con una sola mano, e dalla grande sfera gialla che spicca sul lato destro del dipinto, un luminoso sole assunto a simbolo dei cambiamenti radicali nella società e nella vita quotidiana, nella visione del mondo e nella sua rappresentazione.

Il secondo momento di riflessione, che rappresenta una chiave di lettura per l'opera, è legato al punto interrogativo posto dopo l'espressione 'radioso avvenire'. 'Radioso avvenire' era la parola d'ordine della propaganda sovietica, che chiedeva grandi sacrifici affinché potesse realizzarsi un progetto escatologico che avrebbe abolito le classi sociali, messo fine alle guerre e permesso la nascita di un uomo nuovo. L'espressione 'radioso avvenire' condensa tutta la dimensione utopica del pensiero 
marxista e leninista, ma il punto interrogativo che segue sottende anche il suo fallimento. Cento anni dopo, quale lettura possiamo dare del pensiero utopico che ha alimentato la Rivoluzione d'Ottobre? Come si è articolato? Come hanno contribuito le scienze umane all'elaborazione di percorsi alternativi? Senza pretendere di rispondere in modo esaustivo a queste domande, i contributi della miscellanea propongono alcuni percorsi di studio. Gli autori esaminano varie pratiche artistiche e concezioni teoriche sviluppatesi in un rapporto più o meno diretto con il tema dell'utopia, o concentratesi ad esplorare i complessi legami che uniscono le scienze umane e la politica. Emerge l'annosa questione dell'engagement, l'impegno, da alcuni denigrato come una sottomissione della creazione all'attivismo, da altri sostenuto come un atto di emancipazione. In questa prospettiva, la scelta del titolo Radioso avvenire? non è ingenua: è lungo le direttrici della 'luce' e della 'problematicità' che si sviluppa la riflessione sui cento anni trascorsi dalla Rivoluzione d'Ottobre, analizzando gli effetti immediati e le ripercussioni a lungo termine di uno degli eventi che hanno segnato profondamente la storia mondiale.

Il volume consta di ventinove pregevoli contributi preceduti dall'introduzione dei curatori, preziosa, in quanto offre una panoramica d'insieme sulla pluralità delle voci degli autori, il cui profilo è fornito in appendice. I saggi sono divisi in quattro sezioni tematiche che riflettono i diversi ambiti culturali in cui si sviluppa la riflessione. I nove saggi della prima parte, Cronografie e topografie, presentano la Rivoluzione in una prospettiva storico-politica, spaziale e religiosa. La seconda parte, costituita da otto saggi sul tema della Letteratura, è dedicata ad autori che cercano di sfuggire ai quadri concettuali del pensiero materialista e dell'ideologia socialista, e ad alcune puntuali analisi della ricezione del 1917 da parte della letteratura e della cultura, non solo in Unione Sovietica, ma anche in Occidente e, in particolare, in Italia. La terza sezione sulle Arti visive propone otto saggi che prendono in esame il concetto di utopia applicandolo alla pittura e alla sperimentazione artistica. Infine, la quarta parte, dedicata a Moda e spettacolo raccoglie quattro originali saggi che evidenziano come la Rivoluzione abbia influenzato il mondo della moda e dello spettacolo, non solo in patria, ma anche in Europa, soprattutto in Francia, nella ricerca di nuove forme di estetica e di espressione.

Nell'ampia prospettiva aperta dai contributi presenti nel volume, la scelta del tema 'radioso avvenire?' può, dunque, essere letta come un tentativo di concentrare la discussione sulle sorti di un'utopia che ha toccato ogni ambito delle scienze umane. In molti casi il sistema di propaganda, nel suo desiderio di legittimare il potere e i suoi obiettivi, ha portato a un ideologismo sterile. Nel romanzo Il radioso avvenire di Aleksandr Zinov'ev (al quale è dedicato uno dei saggi), di pari passo col procedere della storia, diverse situazioni ci fanno capire l'ironia di questo slogan, che dovrebbe indicare il percorso della società, ma si rivela sproporzionato rispetto alla realtà in cui evolvono i protagonisti. Nessuno crede veramente in un futuro luminoso, ognuno segue la propria strada, avvilito dai privilegi delle classi sociali superiori, in una società che si suppone senza classi.

Allo stesso modo, nella molteplicità delle aspettative generate dalla rivoluzione politica e delle energie creative liberate dalla rivoluzione artistica e umana, i saggi de Il radioso avvenire?, sembrano confermare il fallimento di una delle più grandi utopie della modernità, trasformando il 'radioso avvenire' in un retaggio del passato. 